\title{
On some properties of the difference spectrum
}

\author{
D. L. Salinger* and J. D. Stegeman
}

\begin{abstract}
We consider problems arising from trying to lift the non-synthesis spectrum of a closed set in a quotient of a locally compact, non-metrizable, abelian group. In particular, we get contrasting results for the difference spectra of linear combinations of elements of a regular Banach function algebra, depending on whether the Gelfand space is metrizable or not.
\end{abstract}

1991 Mathematics Subject Classification: 43A45, 46J10

\section{Conventions and Notation}

Let $A$ be a commutative, semisimple, regular Banach algebra with Gelfand space $X$. We shall identify $A$ with its Gelfand representation, that is, we shall think of $A$ as a Banach algebra of continuous functions (vanishing at infinity) on the locally compact space $X$. We shall assume that the functions in $A$ with compact supports are dense in $A$. Our standard example is the Fourier algebra $A=A(G)=\mathcal{F} L^{1}(\Gamma)$, where $G$ is a locally compact abelian group and $\Gamma$ is its dual group. In practice we often take $G$ compact (hence $\Gamma$ discrete). As our emphasis is on local phenomena, this is not really a loss of generality.

We now introduce notation that will be used throughout. Consider a topological space $X$ and a point $x \in X$. We use the symbols $=_{x}, \epsilon_{x}, \subset_{x}, \ldots$ to denote equality, belonging, inclusion, ..., locally at $x$. Thus, for functions $f$ and $g$ defined on $X$, we say that $f$ and $g$ are locally equal at $x$, and we write $f={ }_{x} g$, if there is a neighbourhood $U$ of $x$ such that $\left.f\right|_{U}=\left.g\right|_{U}$; similarly, two subsets $E$ and $F$ of $X$ are called locally equal at $x$ (which we denote by $E={ }_{x} F$ ) if $E \cap U=F \cap U$ for some neighbourhood $U$ of $x$. We observe that $E={ }_{x} X$ if (and only if) $x$ is an interior point of $E$, while $E={ }_{x} \emptyset$ if $x \notin \bar{E}$, and $\emptyset \neq_{x} E \neq_{x} X$ if $x \in \partial E$. Note that the set of points where two functions, or two subsets, are equal, is always open.

Suppose next that $\Phi$ is a collection of functions defined on $X$ and that $f$ is a

*D.L.Salinger was supported by the British Council - DAAD link between Leeds and Saarbrucken under ARC project 713 . We acknowledge this support with thanks. 
function defined on $X$. We say that $f$ belongs to $\Phi$, locally at $x$, and we write $f \in \epsilon_{x} \Phi$, if there is a function $g \in \Phi$ such that $f={ }_{x} g$. The set

$$
\Delta(f, \Phi):=\{x \in X \mid f \notin x \Phi\}
$$

will be called the spectrum of $f$ with respect to $\Phi$. The spectrum is always a closed set. In particular, the spectrum of $f$ with respect to $O:=\{0\}$ is the support of $f$ (in the usual sense); thus

$$
\operatorname{supp} f=\Delta(f, O)=\left\{x \in X \mid f \neq_{x} 0\right\} .
$$

Further, if $\Phi_{1}$ and $\Phi_{2}$ are two collections of functions defined on $X$, then we say that $\Phi_{1}$ is contained in $\Phi_{2}$, locally at $x$, and we write $\Phi_{1} \subset_{x} \Phi_{2}$, if for each $f \in \Phi_{1}$ we have $f \in_{x} \Phi_{2}$. The two collections are called locally equal at $x$ if $\Phi_{1} \subset_{x} \Phi_{2}$ and $\Phi_{2} \subset_{x} \Phi_{1}$.

If the space $X$ is locally compact, then, in the same way, we define symbols $=_{\infty}, \epsilon_{\infty}, \subset_{\infty}, \ldots$, to indicate validity outside some compact subset of $X$. For instance, $f=\infty 0$ if and only if $f$ has compact support.

We shall have occasion to use a more general form of the above notation. For an arbitrary subset $Y$ of $X$ we shall use symbols $=_{Y}, \in_{Y}, \subset_{Y}, \ldots$, to denote validity of the relation concerned in a neighbourhood of $Y$, and we shall say that the relation holds locally at $Y$. It is evident that if a relation holds locally at some subset $\mathrm{Y}$ of $X$, then, a fortiori, the same relation holds at any subset of $Y$. In the opposite direction we have the following: if $Y \subset X$ and if $f={ }_{x} g$ for all $x \in Y$, then $f==_{Y} g$, and similarly for local equality of two sets $E$ and $F$. However, if $f \in_{Y} \Phi$ and $f \in_{Z} \Phi$ ( $f$ a function, $\Phi$ a collection of functions), it does not follow that $f \in_{Y \cup Z} \Phi$, nor does $\Phi_{1} \subset_{Y} \Phi_{2}$ and $\Phi_{1} \subset_{Z} \quad \Phi_{2}$ imply $\Phi_{1} \subset_{Y \cup Z} \Phi_{2}$. For instance, suppose that $X$ consists of two points. We can then identify the real-valued functions on $X$ with $\mathbb{R}^{2}$. Now take $\Phi_{1}=\{(1,0),(0,1)\}$ and $\Phi_{2}=\{(0,0),(1,0),(0,1),(1,1)\}$. Then $\Phi_{1}={ }_{x} \Phi_{2}$ for all $x \in X$, yet $\Phi_{1}=X \Phi_{2}$ does not hold.

Finally, we shall use $\neq_{x}, \notin_{x}, \not \subset_{x}, \ldots$, to denote that the relation in question does not hold, locally at $x$, and similarly for a subset $Y$ instead of a point $x$.

\section{The difference spectrum}

Now let $A$ be a Banach algebra as described in Section 1. In [2] we gave the following definition: the difference spectrum of two (not necessarily closed) ideals $I_{1}$ and $I_{2}$ in $A$ is the set $\Delta\left(I_{1}, I_{2}\right)$ of all $x \in X$ where $I_{1}$ and $I_{2}$ are locally different; thus

$$
\Delta\left(I_{1}, I_{2}\right)=\left\{x \in X \mid I_{1} \neq{ }_{x} I_{2}\right\} .
$$

If the locally compact space $X$ is not compact, we also define the extended difference spectrum $\Delta_{\infty}\left(I_{1}, I_{2}\right)$ to be the set

$$
\Delta_{\infty}\left(I_{1}, I_{2}\right)=\left\{x \in X \cup\{\infty\} \mid I_{1} \neq_{x} I_{2}\right\} .
$$


A partition-of-unity argument gives $I_{1}=I_{2}$ if (and only if) $\Delta_{\infty}\left(I_{1}, I_{2}\right)=\emptyset$ ([1, p.19]). When $I_{1}$ and $I_{2}$ are closed, we already have $I_{1}=I_{2}$ if $\Delta\left(I_{1}, I_{2}\right)=\emptyset$ (since the functions in $A$ with compact supports are dense, by assumption).

In a completely analogous way the following result is proved. (Note that the assumption that $I$ be an ideal is essential, in view of the remarks towards the end of Section 1.)

Proposition 2.1. Let $A$ and $X$ be as before, and let $Y$ be a compact subset of $X$. Let I be an ideal in $A$, and let $f$ be a function, defined on $X$. If $f \in_{x} I$ for all $x \in Y$, then $f \in \in_{Y} I$.

Let $I$ be an ideal in $A$. Then the hull of $I$ (also called the zero set or the cospectrum of $I)$ is the set hull $(I)=\Delta(I, A)=\{x \in X \mid I \neq x A\}$.

Let $E$ be a closed subset of $X$. The kernel of $E$ is the ideal

$$
I_{E}=\left\{f \in A|f|_{E}=0\right\} .
$$

It is the largest ideal with hull $E$. The smallest ideal with hull $E$ is the ideal

$$
j_{E}=\left\{f \in A \mid f={ }_{E \cup\{\infty\}} 0\right\} .
$$

The closure of $j_{E}$ is denoted by $J_{E}$; it is the smallest closed ideal with hull $E$. As is well-known, $E$ is called a set of (spectral) synthesis if $J_{E}=I_{E}$; otherwise $E$ is called a set of non-synthesis. We denote the difference spectrum $\Delta\left(J_{E}, I_{E}\right)$ by $\Delta_{E}$, and we call this set the spectrum of non-synthesis of $E$.

Note that $\Delta_{E} \subset \partial E$ and that $\Delta_{E}=\emptyset$ if and only if $E$ is a set of synthesis.

We also use the following terminology: the spectrum and the cospectrum of an ideal $I$ are defined as the difference spectra of $I$ and $A$ and of $I$ and $O=\{0\}$, respectively; thus

$$
\begin{aligned}
& \operatorname{cosp}(I)=\Delta(I, A)=\left\{x \in X \mid I \neq_{x} A\right\}, \\
& \operatorname{spec}(I)=\Delta(O, I)=\left\{x \in X \mid O \neq_{x} I\right\},
\end{aligned}
$$

In $[1, \mathrm{p} .125]$ the spectrum of an ideal is defined as the closure of the above set $\operatorname{spec}(I)$; our definition above seems more natural. For instance, the spectrum of $j_{E}$ is the open set $X \backslash E$.

In the special case that $X$ is metrizable, the following holds (cf. [2, p. 532]): if $I_{1}$ is closed, then $\left\{x \in X \mid I_{1} \subset_{x} I_{2}\right)$ is open. As a consequence we have the following result.

Proposition 2.2. Let $A$ and $X$ be as before. Suppose that $X$ is metrizable. Then:

(i) if $I_{1}, I_{2}$ are closed ideals of $A$, then $\Delta\left(I_{1}, I_{2}\right)$ is closed;

(ii) the cospectrum (the hull) of any ideal is closed;

(iii) the spectrum of any closed ideal is closed.

Proof. $\Delta\left(I_{1}, I_{2}\right)$ is the complement of the intersection of two open sets. $\Delta(I, A)$ and $\Delta(O, I)$ are the complements of $\left\{x \in X \mid A \subset_{x} I\right\}$ and $\left\{x \in X \mid I \subset_{x} O\right\}$, respectively. 
Now consider the case where $X$ is non-metrizable. In [2] we have proved the following:

Theorem 2.3. Let $G$ be a non-metrizable compact abelian group (thus $\Gamma$ is a discrete uncountable group).

(i) There is a set of non-synthesis E such that its spectrum of non-synthesis $\Delta_{E}$ is not closed.

(ii) The set $\Delta(O, I)$ (the spectrum of $I$ ) is closed whenever I is a closed ideal.

From Section 1 we recall that the spectrum $\Delta(f, I)$ of an individual function $f \in A$ with respect to an arbitrary ideal $I$ of $A$ is closed, even if $X$ is non-metrizable.

\section{Linear combinations of functions}

Let $A$ and $X$ be as before. Consider a compact non-synthesis subset $E$ of $X$. For $f \in I_{E}$ we shall denote the spectrum $\Delta\left(f, J_{E}\right)$ of $f$ with respect to $J_{E}$, by $\Delta_{f}$, or by $\Delta_{f}^{E}$, if it is necessary to specify the set $E$. For two functions $f, g \in I_{E}$ it is obvious that $\Delta_{f+g} \subset \Delta_{f} \cup \Delta_{g}$. It is also clear that equality need not hold in general: it may happen that part of the 'bad behaviour' of $f$ is neutralized by that of $g$. If this happens, we can avoid it by adding to $f$, not $g$ itself, but a suitable multiple of $g$. The multiple has to be chosen in such a way that no "neutralization of bad behaviour' occurs at some other part of the spectrum. The following lemma shows that suitable multiples are always available when $X$ is metrizable.

Lemma 3.1. Suppose that the Gelfand space $X$ of the Banach algebra $A$ is metrizable. Let $E$ be a compact set of non-synthesis in $X$. Consider two functions $f, g \in$ $I_{E} \backslash J_{E}$. Then there are at most countably many $\alpha \in \mathbb{C}$ such that $\Delta_{f+\alpha g} \neq \Delta_{f} \cup \Delta_{g}$.

Proof. Clearly $\Delta_{f+\alpha g} \subset \Delta_{f} \cup \Delta_{g}$, any $\alpha \in \mathbb{C}$.

Consider the sets $U_{\alpha}, \alpha \in \mathbb{C}$, defined by $U_{\alpha}=\left(\Delta_{f} \cup \Delta_{g}\right) \backslash \Delta_{f+\alpha g}$. Suppose that $x \in U_{\alpha} \cap U_{\beta}$. Then $f+\alpha g \in_{x} J_{E}$ and $f+\beta g \in_{x} J_{E}$, hence (subtract!) $(\alpha-\beta) g \in_{x} J_{E}$ and, similarly, $(\beta-\alpha) f \in_{x} J_{E}$. If $\alpha \neq \beta$, this implies that $x \notin \Delta_{f} \cup \Delta_{g}$, which gives a contradiction. We conclude that the sets $U_{\alpha}(\alpha \in \mathbb{C})$ are disjoint.

It now follows from the metrizability of $X$ that there can be at most countably many $\alpha$ 's for which $U_{\alpha} \neq \varnothing$. Indeed, we can fix a metric $d$ giving the topology on $X$. The sets $U_{\alpha}$ are relatively open in $\Delta_{f} \cup \Delta_{g}$, hence for a point $x_{\alpha} \in U_{\alpha}$ there exists $\delta_{\alpha}>0$ such that, for $y \in \Delta_{f} \cup \Delta_{g}$, we have $d\left(x_{\alpha}, y\right)<\delta_{\alpha} \Rightarrow y \in U_{\alpha}$. If there were uncountably many non-empty $U_{\alpha}$, there would be a $\delta>0$ such that $\delta<\delta_{\alpha}$ for infinitely many distinct values of $\alpha$, giving an infinite set of points $x_{\alpha}$ with no accumulation point in the compact set $\Delta_{f} \cup \Delta_{g}$, which is impossible.

Our aim is to prove that in the case of a metrizable Gelfand space the result of the lemma can be extended to infinite linear combinations. But first we need another lemma. 
Lemma 3.2. Let $A$ and $X$ be as before. Suppose that $X$ is metrizable. Let $E$ be a compact subset of $X$ of non-synthesis with spectrum of non-synthesis $\Delta_{E}$. Then there exists a sequence of functions $\left(\tau_{k}\right)_{k>1}$ in $A$ such that for every $x \in \Delta_{E}$, every neighbourhood $U$ of $x$ and every $n \geq 1$ there is $a k>n$ such that $\tau_{k}={ }_{x} 1$ and $\tau_{k}={ }_{y} 0$ for every $y \notin U$.

Proof. Since $\Delta_{E} \subset E$ is metrizable and compact, we may select a countable collection of open sets $U_{n}, n \in \mathbb{N}$, in $X$, such that $U_{n} \cap \Delta_{E}$ is a basis of open sets in the relative topology on $\Delta_{E}$, each $\bar{U}_{n}$ is compact and for each $x \in U_{n}$, there is an $m$ such that $x \in U_{m} \subset \bar{U}_{m} \subset U_{n}$. It follows from the regularity of $A$ that, given any pair $U_{m}, U_{n}$ such that $\bar{U}_{m} \subset U_{n}$, there exists a function $\tau_{m, n} \in A$ such that $\tau_{m, n}=1$ on $U_{m}$ and $\tau_{m, n}=0$ outside $U_{n}$. The collection of such $\tau_{m, n}$ is countable and we relable it as $\tau_{k}, k \in \mathbb{N}$, in such a way that each $\tau_{m, n}$ occurs infinitely often. The sequence $\tau_{k}$ now has the required properties.

Theorem 3.3. Suppose that the Gelfand space $X$ of the Banach function algebra $A$ is metrizable. Let $E$ be a compact non-synthesis subset of $X$. Let functions $f_{j} \in I_{E} \backslash J_{E}(j \geq 1)$ be given. Then there exist $\alpha_{j} \in \mathbb{C}(j \geq 1)$ such that $g:=\sum_{j=1}^{\infty} \alpha_{j} f_{j}$ exists and

$$
\Delta_{g}=\overline{\bigcup_{j=1}^{\infty} \Delta_{f_{j}} .}
$$

Proof. As in the proof of Lemma 3.1, one inclusion is obvious. As the set $\Delta_{g}$ is closed, it therefore suffices to show that for every $n \geq 1$ one has $\Delta_{f_{n}} \subset \Delta_{g}$. We can further assume, without loss of generality, that $\left\|\bar{f}_{j}\right\|=1$ for all $j \geq 1$.

Take a sequence $\left(\tau_{j}\right)_{j \geq 1}$ as in Lemma 3.2. We shall choose the numbers $\alpha_{k}$ $(k \geq 1)$ inductively, and we shall denote the partial sums $\sum_{j=1}^{k} \alpha_{j} f_{j}$ by $g_{k}$. Take $\alpha_{1}=1$. Suppose that for some $k \geq 2$ the numbers $\alpha_{1}, \ldots, \alpha_{k-1}$ have been chosen. Take $\alpha_{k} \in \mathbb{C} \backslash\{0\}$ such that

$$
\begin{aligned}
& \text { (i) } \Delta_{g_{k}}=\bigcup_{j=1}^{k} \Delta_{f_{j}}, \\
& \text { (ii) }\left|\alpha_{k}\right| \leq\left|\alpha_{k-1}\right| / 2, \\
& \text { (iii) if } g_{k-1} \tau_{k} \notin J_{E} \text {, then }\left|\alpha_{k}\right|<\frac{\operatorname{dist}\left(g_{k-1} \tau_{k}, J_{E}\right)}{2|| \tau_{k}||} \text {, }
\end{aligned}
$$

where, for $f \in A$ and $\Phi \subset A$, we $\operatorname{define} \operatorname{dist}(f, \Phi)=\inf \{\|f-g\| \mid g \in \Phi\}$. It follows from Lemma 3.1 that (i) can be satisfied for arbitrarily small values of $\alpha$. Therefore a choice as above is always possible.

Now take any $x \in \Delta_{f_{n}}$ and suppose that $x \notin \Delta_{g}$. From (i) it follows that $x \in \Delta_{g_{n}}$. Write $U=X \backslash \Delta_{g}$. By Lemma 3.2 , there is a $k>n$ such that $\tau_{k}={ }_{x} 1$ and $\tau_{k}={ }_{y} 0$ for every $y \notin U$. If $y \in U$ then $y \notin \Delta_{g}$, hence $g \in_{y} J_{E}$, hence also $g \tau_{k} \in_{y} J_{E}$. If $y \notin U$ then $g \tau_{k}={ }_{y} 0 \in J_{E}$. It follows that $\Delta_{g \tau_{k}}=\varnothing$, hence $g \tau_{k} \in J_{E}$. 
On the other hand, $\Delta_{f_{n}} \subset \Delta_{g_{k-1}}$ (by (i)), so $g_{k-1} \tau_{k}={ }_{x} g_{k-1} \notin_{x} J_{E}$, and hence $g_{k-1} \tau_{k} \notin J_{E}$. We have

$\left\|g \tau_{k}-g_{k-1} \tau_{k}\right\|=\left\|\sum_{j=k}^{\infty} \alpha_{j} f_{j} \tau_{k}\right\| \leq \sum_{j=k}^{\infty}\left|\alpha_{j}\right|\left\|\tau_{k}\right\| \leq 2\left|\alpha_{k}\right|\left\|\tau_{k}\right\|<\operatorname{dist}\left(g_{k-1} \tau_{k}, J_{E}\right)$, from (ii) and (iii) above. It follows that $g \tau_{k} \notin J_{E}$, a contradiction.

We now give an example showing that the metrizability of the space $X$ is crucial.

Proposition 3.4. There exists a compact non-metrizable abelian group $G$ (i.e., the dual group $\Gamma$ is discrete and uncountable), a closed set $E \subset G$ of non-synthesis, and functions $f, g \in I_{E} \backslash J_{E}$ such that $\Delta_{f+\alpha g} \neq \Delta_{f} \cup \Delta_{g}$ for all $\alpha \in \mathbb{C}$ with $|\alpha| \leq 1$.

Proof. Denote the set $\{\alpha \in \mathbb{C}|| \alpha \mid \leq 1\}$ by $D$. We need the following three ingredients:

(i) A function $\varphi \in A\left(\mathbb{T}^{2}\right)$ with range $(\varphi)=D$ : for example $\varphi(x, y)=\mathrm{e}^{2 \pi \mathrm{i} x} \cos 2 \pi y$.

(ii) A set of non-synthesis $F \subset \mathbb{T}$ and a function $\psi \in I_{F} \backslash J_{F}$; we may assume that $\Delta_{\psi}^{F}=\Delta_{F}=F$.

(iii) A non-metrizable compact abelian group $H$. We take

$$
H=\prod_{(x, y) \in \mathbb{T}^{2}}\{0,1\}=\left\{\left(\varepsilon_{x, y}\right) \mid \varepsilon_{x, y} \in\{0,1\} \quad\left((x, y) \in \mathbb{T}^{2}\right)\right\} .
$$

(the corners of an uncountable-dimensional cube). The zero element is $\underline{0}=(0)_{x, y}$. The elements $p_{x, y}=\left(\varepsilon_{x^{\prime}, y^{\prime}}\right)$ with $\varepsilon_{x, y}=1, \varepsilon_{x^{\prime}, y^{\prime}}=0$ otherwise, constitute the set $C_{1}$ of all those corners of the cube which are adjacent to $\underline{0}$.

Take $G=\mathbb{T}^{2} \times \mathbb{T} \times H$, and consider the subset $\widetilde{F}=\{(0,0)\} \times F \times\{\underline{0}\}$ of $G$. By the injection theorem for sets of synthesis $([1$, p.149]) $\widetilde{F}$ is a subset of non-synthesis of $G$.

For each $(x, y) \in \mathbb{T}^{2}$ we now consider the set $E_{x, y}=\{(x, y)\} \times F \times\left\{p_{x, y}\right\} \subset G$. All $E_{x, y}$ are translates of $\tilde{F}$, hence they are sets of non-synthesis in $G$ as well. Let $E$ be the closure of the union of these sets. Then it is not hard to see that

$$
E=\left(\bigcup_{(x, y) \in \mathbb{T}^{2}}\{(x, y)\} \times F \times\left\{p_{x, y}\right\}\right) \cup\left(\mathbb{T}^{2} \times F \times\{\underline{0}\}\right) .
$$

Now define $f, g \in I_{E}$ by $f(x, y, z, t)=-\varphi(x, y) \psi(z)$ and $g(x, y, z, t)=\psi(z)$, where $(x, y, z) \in \mathbb{T}^{3}$ and $t \in H$. It is easily seen that $\Delta_{g}=E$ and that $\Delta_{f} \subset \Delta_{g}$, thus $\Delta_{f} \cup \Delta_{g}=\Delta_{g}$; here $\Delta=\Delta^{E}$. Moreover, we have $(f+\alpha g)(x, y, z, t)=$ $(\alpha-\varphi(x, y)) \psi(z)$. For $(x, y)$ such that $\varphi(x, y)=\alpha$ the function $f+\alpha g$ vanishes on $\{(x, y)\} \times \mathbb{T} \times\left\{p_{x, y}\right\}$, a set of synthesis. Therefore, $\Delta_{f+a g} \cap E_{x, y}=\emptyset$ (when $\varphi(x, y)=\alpha)$, and hence $\Delta_{f+a g} \neq \Delta_{f} \cup \Delta_{g}$. 


\section{Lifting the spectrum of non-synthesis}

Let $G$ be a compact abelian group and let $H$ be a closed subgroup. We write $\pi$ for the canonical mapping $\pi: G \rightarrow G / H$. Let $E$ be a closed subset of $G / H$. In [1, p.145] it is shown that $E$ is a set of non-synthesis for $G / H$ if and only if $\pi^{-1} E$ is a set of non-synthesis for $A(G)$. Does this result extend to the non-synthesis spectrum?

It is not difficult to adapt Reiter's method to show that $\Delta_{\pi^{-1} E} \supset \pi^{-1} \Delta_{E}$ [2]. But trying to prove the opposite inclusion meets with difficulties, which we now describe. (It was an attempt to circumvent those difficulties which led to the results of the previous section.)

First we discuss a natural way to write any function $f \in A(G)$ as a sum of functions in $A(G)$, each of which is the product of a character by a function that is constant on cosets of $H$.

Lemma 4.1. For every $f \in A(G)$ there is a countable (possibly finite) set $R \subset \Gamma$ such that each coset of $H^{\perp}$ contains at most one element of $R$ and such that for each $\chi \in R$ there is a function $f_{\chi} \in A(G / H)$ such that

$$
f=\sum_{\chi \in R} \chi \cdot f_{\chi} \circ \pi
$$

Proof. For $f \in A(G)$, we have $f(x)=\sum_{\gamma \in \Gamma} \hat{f}(\gamma)\langle x, \gamma\rangle$ with $\sum_{\gamma \in \Gamma}|\hat{f}(\gamma)|<\infty$. Consider the cosets of $H^{\perp}$ in $\Gamma$. At most countably many of them contain at least one element $\gamma$ for which $\hat{f}(\gamma) \neq 0$. Take a set $R \subset \Gamma$ such that each of these cosets contains exactly one element of $R$ (such a set $R$ is called a set of representatives for these cosets). Then

$$
f(x)=\sum_{\chi \in R} \sum_{\gamma \in H^{\perp}} \hat{f}(\gamma+\chi)\langle x, \gamma+\chi\rangle=\sum_{\chi \in R}\langle x, \chi\rangle \sum_{\gamma \in H^{\perp}} \hat{f}(\gamma+\chi)\langle\pi(x), \gamma\rangle .
$$

Identifying $H^{\perp}$ with the dual of $G / H$, and defining, for arbitrary $\chi \in \Gamma$, functions $f_{\chi} \in A(G / H)$ by $f_{\chi}=\sum_{\gamma \in H^{\perp}} \hat{f}(\gamma+\chi) \gamma$, we obtain $f=\sum_{\chi \in R} \chi \cdot f_{\chi} \circ \pi$, as desired. $\square$

Now take $f \in I_{\pi^{-1} E}$. Consider any $y \in \pi^{-1} E$. We have then, for arbitrary $z \in H, y+z \in \pi^{-1} E$ as well. Writing $f$ as in Lemma 4.1 we obtain, for arbitrary $z \in H$

$$
0=f(y+z)=\sum_{\chi \in R}\left(\langle y, \chi\rangle \cdot f_{\chi}(\pi(y))\right)\langle z, \chi\rangle
$$

This is the Fourier series for the function $z \mapsto f(y+z)$, which in this case is the zero function. Therefore all its Fourier coefficients are zero. We conclude that the functions $f_{\chi}$ in the above proof satisfy $f_{\chi}(\pi(y))=0$ for all $y \in \pi^{-1} E$ : thus $f_{\chi} \in I_{E}$. 
For $w \notin \Delta_{E}$ we know that, for all $\chi \in R$, there exists a neighbourhood $U_{\chi}$ of $w$ such that $f_{\chi} \in_{U_{\chi}} J_{E}$. In the case where $G / H$ is metrizable, we can choose $U_{\chi}$ independently of $\chi$ and conclude the proof. We do not know how to do this for the general non-metrizable case. On the other hand, if we knew it failed in a specific case, then we would have an example where $\Delta_{\pi^{-1} E} \neq \pi^{-1} \Delta_{E}$, as the following result shows.

Proposition 4.2. Let $G_{0}$ be a compact abelian group and let $E$ be a closed subset of $G_{0}$. Let $w \in E$ be such that there is a sequence $\left(f_{n}\right)_{n \geq 1}$ in $I_{E}$ such that for all neighbourhoods $U$ of $w$ there is an $n$ such that $f_{n} \notin_{U} J_{E}$. Then there is an element $t \in \mathbb{T}$ such that $(w, t) \in \Delta_{E \times \mathbb{T}}$.

Proof. Suppose there exists no $t \in \mathbb{T}$ such that $(w, t) \in \Delta_{E \times \mathbb{T}}$. We may assume that $\left\|f_{n}\right\|_{A\left(G_{0}\right)}=2^{-n}(n \geq 1)$. Let $f \in I_{E \times \mathbb{T}}$ be defined by

$$
f(x, t)=\sum_{n=1}^{\infty} f_{n}(x) e^{i n t},
$$

for $x \in G_{0}$ and $t \in \mathbb{T} \cong[-\pi, \pi[$.

Then, for each $t \in \mathbb{T}$ there exist a function $g_{t} \in J_{E \times \mathbb{T}}$ and open neighbourhoods $U_{t}$ of $w$ in $G_{0}$ and $V_{t}$ of $t$ in $\mathbb{T}$ such that $f=g_{t}$ on $U_{t} \times V_{t}$. Now choose a finite subcover $V_{t_{1}}, \ldots V_{t_{n}}$ of $\mathbb{T}$ : we denote the intersection of the corresponding $U_{t_{j}}$ by $U$, and we write $g_{k}$ instead of $g_{t_{k}}$.

Let $W_{1}, \ldots, W_{n}$ be an open cover of $\mathbb{T}$ such that $W_{k} \subset \bar{W}_{k} \subset V_{t_{k}}$, for $k=$ $1, \ldots, n$. Let $\sigma_{k}$, for $k=1, \ldots, n$, be functions in $A(\mathbb{T})$ such that $\sigma_{k}(t) \geq 0$ for all $t, \sigma_{k}(t)>0$ when $t \in \bar{W}_{k}$ and supp $\sigma_{k} \subset V_{t_{k}}$. Define $\tilde{\sigma}_{k} \in A\left(G_{0} \times \mathbb{T}\right)$ by $\tilde{\sigma}_{k}(x, t)=\sigma_{k}(t)$. Set

$$
g=\left(\sum_{k=1}^{n} \tilde{\sigma}_{k} g_{k}\right)\left(\sum_{k=1}^{n} \tilde{\sigma}_{k}\right)^{-1} .
$$

$\left(\sum \tilde{\sigma}_{k}>0\right.$ on $G_{0} \times \mathbb{T}$, so, by Wiener's theorem, $\left.\left(\sum_{k=1}^{n} \widetilde{\sigma}_{k}\right)^{-1} \in A\left(G_{0} \times \mathbb{T}\right)\right)$. Then $g \in J_{E \times \mathbb{T}}$ and $g=f$ on $U \times \mathbb{T}$.

To finish the proof, we must show that this leads to a contradiction. Let $\psi \in$ $A\left(G_{0}\right)$ be such that supp $\psi \subset U$ and such that there exists an open neighbourhood $U^{\prime}$ of $w$ with $\psi=1$ on $U^{\prime}$. We may also assume that $0 \leq \psi \leq 1$. Define $\tilde{\psi} \in A\left(G_{0} \times \mathbb{T}\right)$ by $\tilde{\psi}(x, t)=\psi(x)$. Then $g \tilde{\psi}=f \tilde{\psi} \in J_{E \times \mathbb{T}}$.

This means that, given $\varepsilon>0$, there exists an open neighbourhood $N$ of $E \times \mathbb{T}$ with a function $h \in A\left(G_{0} \times \mathbb{T}\right)$ which is identically zero on $N$ and such that $\|h-f \tilde{\psi}\|_{A}<\epsilon$. We can write $h(x, t)=\sum h_{n}(x, t) e^{i n t}$, where $\sum\left\|h_{n}\right\|_{A\left(G_{0}\right)}=$ $\|h\|_{A\left(G_{0} \times \mathbb{T}\right)}$. By looking at the Fourier transforms, we see that $\left\|h_{n}-f_{n} \psi\right\|_{A\left(G_{0}\right)}<$ $\varepsilon$, for $n \geq 1$.

Let $\bar{M}$ be a neighbourhood of $E$ such that $M \times \mathbb{T} \subset N$. For all $t \in \mathbb{T}$, and each fixed $x \in M$, we have $\sum h_{n}(x) e^{i n t}=0$. Hence, by the uniqueness theorem for Fourier series, each $h_{n}(x)=0$ on $M$.

Since $\varepsilon>0$ is arbitrary, we conclude that $f_{n} \psi \in J_{E}$ and thus $f_{n} \in U^{\prime} J_{E}$, for every $n \geq 1$, contrary to the hypotheses. 
To get an example where $\Delta_{E}$ does not lift, it thus suffices to find a compact nonmetrizable abelian group $G_{0}$, with $E$ and $w$ as in the proposition but satisfying, in addition, the condition $w \notin \Delta_{E}$. However, can these two requirements be met simultaneously? As our construction in [2] suggests, a set $E \subset G$ for which $\Delta_{E}$ is not closed may have to be extremely thin (in some sense) at every point $w \in \overline{\Delta_{E}} \backslash \Delta_{E}$, which might be an obstruction for the construction of a sequence $\left(f_{n}\right)_{n \geq 1}$ as in Proposition 4.2 .

So far we have not been able to settle this problem.

\section{References}

[1] H, Reiter, Classical Harmonic Analysis and Locally Compact Groups. Oxford University Press 1968.

[2] D.L. Salinger, J.D. Stegeman, Difference spectra of ideals for non-metrizable groups. J. London Math. Soc. (2), 26, 531-540 (1982). 